January 18, 2010

FERMILAB-PUB-10-009-T

Revised May 26, 2010

\title{
Uplifted supersymmetric Higgs region
}

\author{
Bogdan A. Dobrescu and Patrick J. Fox \\ Theoretical Physics Department, Fermilab, Batavia, IL 60510, USA
}

\begin{abstract}
We show that the parameter space of the Minimal Supersymmetric Standard Model includes a region where the down-type fermion masses are generated by the loop-induced couplings to the up-type Higgs doublet. In this region the downtype Higgs doublet does not acquire a vacuum expectation value at tree level, and has sizable couplings in the superpotential to the tau leptons and bottom quarks. Besides a light standard-like Higgs boson, the Higgs spectrum includes the nearly degenerate states of a heavy spin-0 doublet which can be produced through their couplings to the $b$ quark and decay predominantly into $\tau^{+} \tau^{-}$or $\tau \nu$.
\end{abstract}

\section{Introduction}

The simplest supersymmetrization of the standard model (i.e., the MSSM [1]) involves two Higgs doublets, and the requirement of holomorphy enforces that one of them couples exclusively to the up-type quarks while the other one couples only to down-type quarks and leptons. This implies that both Higgs doublets must get nonzero vacuum expectation values whose ratio $\left\langle H_{u}\right\rangle /\left\langle H_{d}\right\rangle \equiv \tan \beta$ determines by how much the supersymmetric Yukawa couplings differ from those in the standard model. The requirement that these couplings are perturbative up to the Planck scale leads to the standard bounds $2 \lesssim \tan \beta \lesssim 50$

We show here, however, that the MSSM is a viable theory even when $\tan \beta \gg 50$. The down-type quarks and leptons in that case must acquire masses from Yukawa couplings to $H_{u}$. Although these are forbidden by holomorphy, they are generated at 1-loop level once supersymmetry is broken. The Yukawa couplings of $H_{d}$ to the down-type quarks and leptons take values different from those in the usual MSSM, but they do not need to be larger than $O(1)$ despite the size of $\tan \beta$. 
In this region of parameter space, which we dub "uplifted supersymmetry", $\tan \beta$ is not the correct variable to consider when discussing couplings involving quarks or leptons, but does provide a reasonable description in the Higgs-gauge sector. Uplifted supersymmetry has very different Higgs-sector phenomenology from the usual MSSM, with the largest effects occuring for the heavy Higgs states of the MSSM. Since the Yukawa couplings for the down-type quarks and leptons are different from those in the standard model, the production rates of the Higgs states at colliders are modified. Furthermore, the loop generation of the couplings of $H_{u}$ to down-type quarks and leptons means that ratios of $H_{d}$ Yukawa couplings for these fermions are no longer the same as in the standard model or the usual MSSM, leading to peculiar Higgs branching fractions.

Loop corrections to Yukawa couplings in the MSSM have been previously discussed [2]. However, the possibility of $\tan \beta \gg 50$ has been pointed out only in Ref. [3], in the context of up-down Yukawa unification. Some aspects of Higgs phenomenology within the MSSM with $\tan \beta$ as large as 130 have been studied in Ref. [4]. A related theory where all down-type fermions get masses at 1-loop while the up-type quark masses arise at tree level has been investigated in Ref. [5], but loops there involve fields beyond the MSSM.

In Section 2 we first describe the MSSM in the uplifted region at tree-level, where only $H_{u}$ acquires a VEV. We then compute the loop corrections induced by MSSM fields below the supersymmetry breaking scale, which allow $H_{d}$ to acquire a small VEV. This $\mathrm{VEV}$ is insufficient to generate the down-type quark and lepton masses while keeping their Yukawa couplings perturbative. We show in Sections 3 and 4 how loop corrections generate couplings to $H_{u}$ and lead to the correct lepton and down-type quark masses. In Section 5 we discuss the properties of the Higgs states in uplifted supersymmetry. Finally, in Section 6 we mention some phenomenological implications.

\section{Uplifted supersymmetry}

The field content of the uplifted Higgs model considered here is identical to that of the MSSM [1]. The superpotential is exactly as in the usual R-parity conserving MSSM:

$$
W=y_{u} \hat{u}^{c} \hat{Q} \hat{H}_{u}-y_{d} \hat{d}^{c} \hat{Q} \hat{H}_{d}-y_{\ell} \hat{e}^{c} \hat{L} \hat{H}_{d}+\mu \hat{H}_{u} \hat{H}_{d}
$$

where a hat denotes the chiral superfield associated with the corresponding standard model field, and a generation index is implicit. The Yukawa coupling matrix of the uptype quarks, $y_{u}$, is the same as in the standard model. For down-type quarks or leptons, 
the Yukawa couplings $y_{d}$ and $y_{\ell}$ have values different than in the MSSM, as explained later in this section.

\subsection{Tree-level MSSM in the uplifted region}

We assign R-charges such that the soft supersymmetry-breaking term $H_{u} H_{d}$ is forbidden, for example $R\left[\hat{H}_{d}, \hat{Q}, \hat{u}^{c}, \hat{e}^{c}\right]=0$ and $R\left[\hat{H}_{u}, \hat{d}^{c}, \hat{L}\right]=2$. Thus, the Higgs potential is

$$
\left(|\mu|^{2}+m_{H_{u}}^{2}\right)\left|H_{u}\right|^{2}+\left(|\mu|^{2}+m_{H_{d}}^{2}\right)\left|H_{d}\right|^{2}+\frac{g^{\prime 2}}{8}\left(\left|H_{u}\right|^{2}-\left|H_{d}\right|^{2}\right)^{2}+\frac{g^{2}}{2}\left|H_{u}^{\dagger} T^{a} H_{u}+H_{d}^{\dagger} T^{a} H_{d}\right|^{2},
$$

where $m_{H_{u}}^{2}$ and $m_{H_{d}}^{2}$ are supersymmetry-breaking mass-squared parameters, and $T^{a}$ are the $S U(2)_{W}$ generators. We assume that

$$
\begin{aligned}
& |\mu|^{2}+m_{H_{u}}^{2}<0, \\
& |\mu|^{2}+m_{H_{d}}^{2}>0
\end{aligned}
$$

and, in order for the potential to be bounded from below, that

$$
2|\mu|^{2}+m_{H_{u}}^{2}+m_{H_{d}}^{2}>0
$$

This results in only $H_{u}$ acquiring a VEV: $v_{u} \approx 174 \mathrm{GeV}$. Thus, the Higgs boson $h^{0}$ that couples to $W W$ is at tree level entirely part of the $H_{u}$ doublet, and has a squared mass

$$
M_{h^{0}}^{2}=-2\left(|\mu|^{2}+m_{H_{u}}^{2}\right)=M_{Z}^{2}
$$

The other physical states, $H^{0}, A^{0}$ and $H^{ \pm}$, are all part of the $H_{d}$ doublet and have tree-level masses:

$$
\begin{gathered}
M_{H^{0}}^{2}=M_{A^{0}}^{2}=2|\mu|^{2}+m_{H_{u}}^{2}+m_{H_{d}}^{2}, \\
M_{H^{ \pm}}^{2}=M_{A^{0}}^{2}+M_{W}^{2} .
\end{gathered}
$$

Given that $H_{d}$ has no VEV, the down-type quarks and leptons do not acquire masses from the Yukawa couplings given in (2.1). It is at this stage that one would naively dismiss this model. However, the Yukawa couplings (2.1) explicitly break the chiral symmetries from $U(3)^{5}$ to $U(1)_{B} \times U(1)_{L}$, so at some loop level masses will be generated for the downtype quarks and leptons. We will demonstrate that these masses are generated at 1-loop once supersymmetry is broken. This opens up a previously ignored region of parameter space in the MSSM. 


\section{$2.2 \quad$ Effective couplings}

With unbroken supersymmetry, holomorphy dictates that the only allowed Higgs couplings are those derived from the superpotential (2.1). However, once supersymmetry (and the R-symmetry) is broken, all gauge invariant operators may be present in the lowenergy effective Lagrangian. Of most interest to us are those that couple the $H_{u}$ Higgs doublet to down-type quarks and leptons

$$
-y_{d}^{\prime} d^{c} H_{u}^{\dagger} Q-y_{\ell}^{\prime} e^{c} H_{u}^{\dagger} L+\text { H.c. }
$$

In order to identify the diagrams responsible for these effective Yukawa couplings, let us first display the couplings of $H_{u}^{\dagger}$ relevant for this problem. The $F$ term for $H_{d}$ which follows from the superpotential (2.1) is

$$
F_{H_{d}}^{\dagger}=y_{d} \tilde{d}^{c} \widetilde{Q}+y_{\ell} \tilde{e}^{c} \widetilde{L}-\mu H_{u}
$$

This $F$ term generates the following trilinear scalar interactions in the Lagrangian:

$$
\mu^{*} H_{u}^{\dagger}\left(y_{d} \tilde{d}^{c} \widetilde{Q}+y_{\ell} \tilde{e}^{c} \widetilde{L}\right)+\text { H.c. }
$$

In general the gaugino mass terms may be complex, but we choose to work in a basis where the gaugino masses are real and positive and thus appear in the Lagrangian as,

$$
-M_{\widetilde{B}} \widetilde{B} \widetilde{B}-M_{\widetilde{W}} \widetilde{W} \widetilde{W}-M_{\tilde{g}} \tilde{g} \tilde{g}
$$

$H_{u}^{\dagger}$ has couplings to a Higgsino and a wino or a bino, these couplings now include some complex phases $\theta_{W}$ and $\theta_{B}$ :

$$
-\sqrt{2}\left(g e^{-i \frac{\theta_{W}}{2}} H_{u}^{\dagger} T^{a} \widetilde{H}_{u} \widetilde{W}^{a}+\frac{g^{\prime}}{2} e^{-i \frac{\theta_{B}}{2}} H_{u}^{\dagger} \widetilde{H}_{u} \widetilde{B}\right)+\text { H.c. }
$$

Finally, there is a trilinear supersymmetry-breaking term involving $H_{u}$ :

$$
-A_{u} \tilde{u}^{c} \widetilde{Q} H_{u}+\text { H.c. , }
$$

where $A_{u}$ is a mass parameter, and again a generation index is implicit. We will compute the $y_{\ell}^{\prime}$ and $y_{d}^{\prime}$ effective couplings in Sections 3 and 4, respectively.

Another effective coupling in the Lagrangian generated by loops is

$$
-b H_{u} H_{d}+\text { H.c. , }
$$




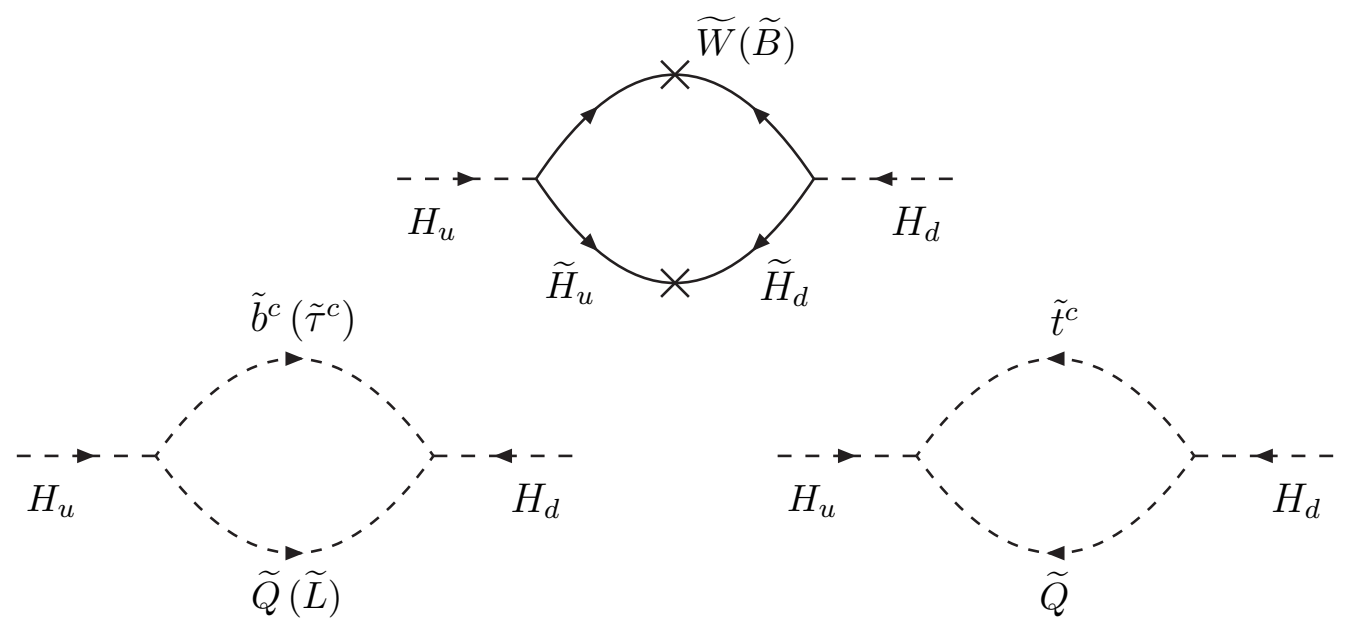

Figure 1: The diagrams responsible for generation of the $H_{u} H_{d}$ soft term.

where $b$ is an induced parameter of mass dimension +2 . This soft supersymmetry-breaking term (sometimes called the $B \mu$ term) is protected by both the R- and PQ symmetries. The latter is broken explicitly by the $\mu$ term, so the loops must involve an insertion of both the $\mu$ term and a gaugino mass or an $A$ term, as in Figure 1. Assuming that the $A$ terms are approximately flavor diagonal and that those for the second or first generations are not much larger than for the third generation, only the third generation $A$ terms may have large contributions to $b$. The couplings of $H_{d}$ relevant here are similar to those for $H_{u}$ given in Eqs. (2.9) $-(2.12)$ :

$$
\left[y_{t}^{*} \mu \tilde{t}^{c \dagger} \widetilde{Q}^{\dagger}-\sqrt{2}\left(g e^{i \frac{\theta_{W}}{2}} \overline{\widetilde{W}^{a}} \widetilde{\widetilde{H}}_{d} T^{a}-\frac{g^{\prime}}{2} e^{i \frac{\theta_{B}}{2}} \overline{\widetilde{B}} \widetilde{\widetilde{H}}_{d}\right)+A_{b} \tilde{b}^{c} \widetilde{Q}+A_{\tau} \tilde{\tau}^{c} \widetilde{L}\right] H_{d}+\text { H.c. }
$$

The diagrams in Figure 1 lare logarithmically divergent and lead to the following expression for $b$ at low energies:

$$
\begin{aligned}
b= & -\frac{\alpha \mu}{2 \pi}\left[\frac{3}{s_{W}^{2}} M_{\tilde{W}} G\left(|\mu|, M_{\tilde{W}}\right) e^{i \theta_{W}}+\frac{1}{c_{W}^{2}} M_{\tilde{B}} G\left(|\mu|, M_{\tilde{B}}\right) e^{i \theta_{B}}\right] \\
& -\frac{\mu}{8 \pi^{2}}\left[3 y_{b}^{*} A_{b} G\left(M_{\tilde{Q}}, M_{\tilde{b}}\right)+y_{\tau}^{*} A_{\tau} G\left(M_{\tilde{L}}, M_{\tilde{\tau}}\right)+3 y_{t}^{*} A_{t} G\left(M_{\tilde{Q}}, M_{\tilde{t}}\right)\right],
\end{aligned}
$$

where $\alpha \approx 1 / 127.9, s_{W}^{2} \approx 0.231, c_{W}^{2}=1-s_{W}^{2}$, and we have defined a logarithmically divergent function

$$
G\left(m_{1}, m_{2}\right)=\frac{1}{m_{2}^{2}-m_{1}^{2}}\left(m_{2}^{2} \ln \frac{\Lambda}{m_{2}}-m_{1}^{2} \ln \frac{\Lambda}{m_{1}}\right) .
$$

The divergence is cutoff at the scale, $\Lambda$, where the soft supersymmetry-breaking terms are generated. We assume that at this scale $b=0$, so that the value (2.15) of $b$ at low 
energies arises through loops involving the MSSM fields between $\Lambda$ and the weak scale. Note that the complex phase of $b$ from Eq. (2.15) may be absorbed by a field redefinition. The resulting VEV for $H_{d}, v_{d}$, depends on the size of the effective Higgs soft mass $m_{H_{d}}$, or alternatively on the mass of $A^{0}$ [see Eq. (2.6)]. At the weak scale this gives the ratio

$$
\frac{v_{u}}{v_{d}} \equiv \tan \beta \approx \frac{1}{|b|} M_{A^{0}}^{2}\left[1+O\left(1 / \tan ^{2} \beta\right)\right] \gg 1
$$

As a numerical example, let us consider the unification relation between the wino and bino masses

$$
M_{\tilde{W}} \approx \frac{3 c_{W}^{2}}{5 s_{W}^{2}} M_{\tilde{B}},
$$

neglect the $A$ terms and the complex phases, and use a cutoff scale of $\Lambda=O(100 \mathrm{TeV})$, as in gauge mediated supersymmetry breaking [6]. For $M_{\tilde{B}}=100 \mathrm{GeV}, M_{A^{0}}=700 \mathrm{GeV}$ and $\mu$ ranging between 100 and $300 \mathrm{GeV}$, we find that $\tan \beta$ varies from 241 to 88. Such large values of $\tan \beta$ are consistent with the perturbativity of the Yukawa couplings because in this uplifted region the down-type fermion masses are generated predominantly by the effective couplings in Eq. (2.7), and only to a small extent by the $H_{d} \mathrm{VEV}$.

Smaller values of $\tan \beta$ are possible, for instance if $M_{A^{0}}$ is smaller. For $\tan \beta \lesssim 50$ one departs from the uplifted region and recovers the usual MSSM, where the down-type masses are generated mainly from their tree-level couplings to the $H_{d} \mathrm{VEV}$. Values of $\tan \beta$ much larger than a few hundred could also occur, even without increasing $M_{A^{0}}$, if there is some cancellation between a small tree-level contribution to $b$ and the loop-induced one, or between the $A$-term and gaugino contributions in Eq. (2.15).

Besides generating a small VEV for $H_{d}$, the loop-induced $H_{u} H_{d}$ term mixes slightly the $h^{0}$ and $H^{0}$ bosons, and shifts their masses, as discussed later in Section 5 .

\section{Loop-induced lepton masses}

There are two types of diagrams contributing to the $y_{\ell}^{\prime}$ Yukawa coupling of $H_{u}^{\dagger}$ to leptons, defined in Eq. (2.7). The first type involves the gaugino interactions of Eq. (2.11), a Higgsino mass insertion, and a wino or bino exchange (see the first two diagrams of Figure 2). The second one arises from the F-term interaction for leptons given in Eq. (2.9)

and a bino mass insertion (last diagram of Figure 21). The gaugino mass insertions are necessary to break the R-symmetry. These 1-loop diagrams are finite, and give rise to an 

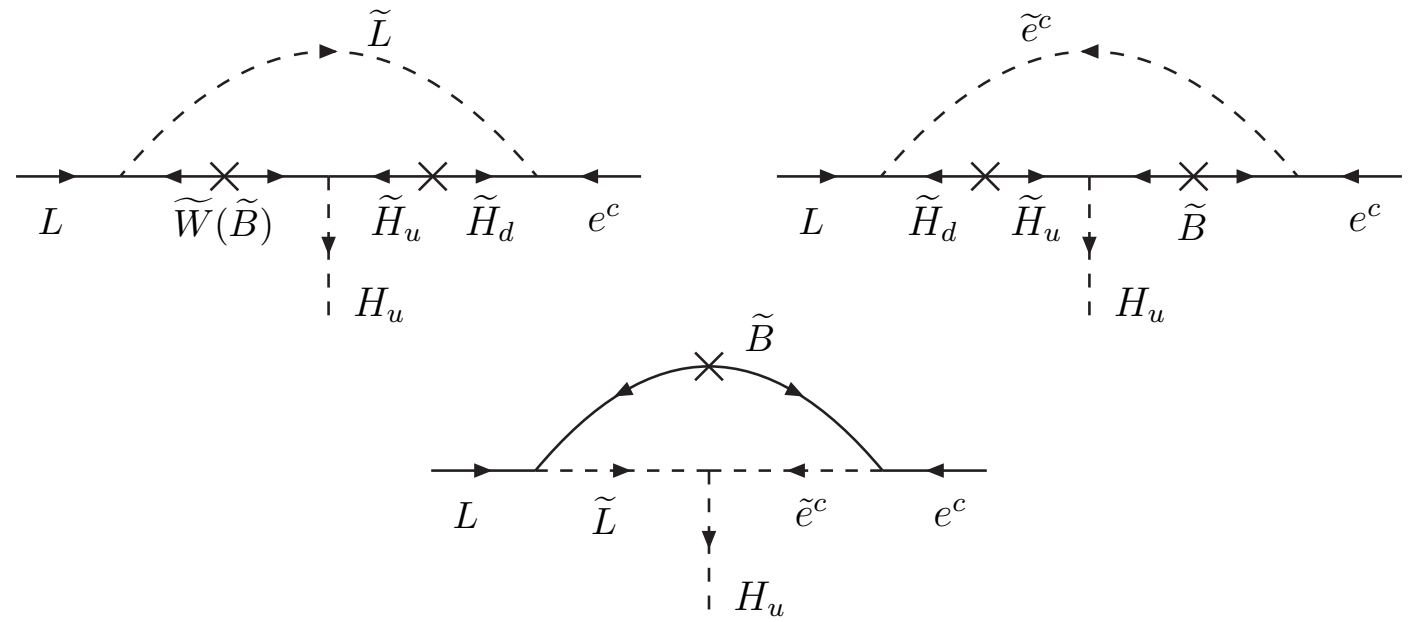

Figure 2: Diagrams responsible for the charged lepton masses. The $\times$ represents a mass insertion. The first two diagrams involve the gaugino interactions of $H_{u}$ given in Eq. (2.11), while the last diagram involves the $F$-term interaction of $H_{u}$ given in Eq. (2.9).

uplifted-Higgs lepton coupling given by (see Appendix)

$$
\begin{aligned}
y_{\ell}^{\prime}=\frac{y_{\ell} \alpha}{8 \pi} e^{-i\left(\theta_{W}+\theta_{\mu}\right)}\left\{-\frac{3}{s_{W}^{2}} F\left(\frac{M_{\tilde{W}}}{M_{\tilde{L}}}, \frac{|\mu|}{M_{\tilde{L}}}\right)+\frac{e^{i\left(\theta_{W}-\theta_{B}\right)}}{c_{W}^{2}}\left[F\left(\frac{M_{\tilde{B}}}{M_{\tilde{L}}}, \frac{|\mu|}{M_{\tilde{L}}}\right)\right.\right. \\
\left.\left.-2 F\left(\frac{M_{\tilde{B}}}{M_{\tilde{e}}}, \frac{|\mu|}{M_{\tilde{e}}}\right)+\frac{2|\mu|}{M_{\tilde{e}}} F\left(\frac{M_{\tilde{B}}}{M_{\tilde{L}}}, \frac{M_{\tilde{e}}}{M_{\tilde{L}}}\right)\right]\right\} .
\end{aligned}
$$

The first term, which is due to wino exchange in the first diagram of Figure 2, usually dominates, but the last two terms (which represent the second and third diagrams) may also be numerically important.

We defined a function of two variables:

$$
F(x, y)=\frac{2 x y}{x^{2}-y^{2}}\left(\frac{y^{2} \ln y}{1-y^{2}}-\frac{x^{2} \ln x}{1-x^{2}}\right) .
$$

Note that this function is well defined for all $x, y>0$; in particular

$$
\begin{aligned}
& F(x, x)=-\frac{x^{2}}{1-x^{2}}\left(1+\frac{2 \ln x}{1-x^{2}}\right), \\
& F(x, 1)=F(1, x)=\frac{x}{1-x^{2}}\left(1+\frac{2 x^{2} \ln x}{1-x^{2}}\right),
\end{aligned}
$$

and $F(1,1)=1 / 2$. For any $x, y>0$, the function satisfies

$$
0<F(x, y)<1
$$




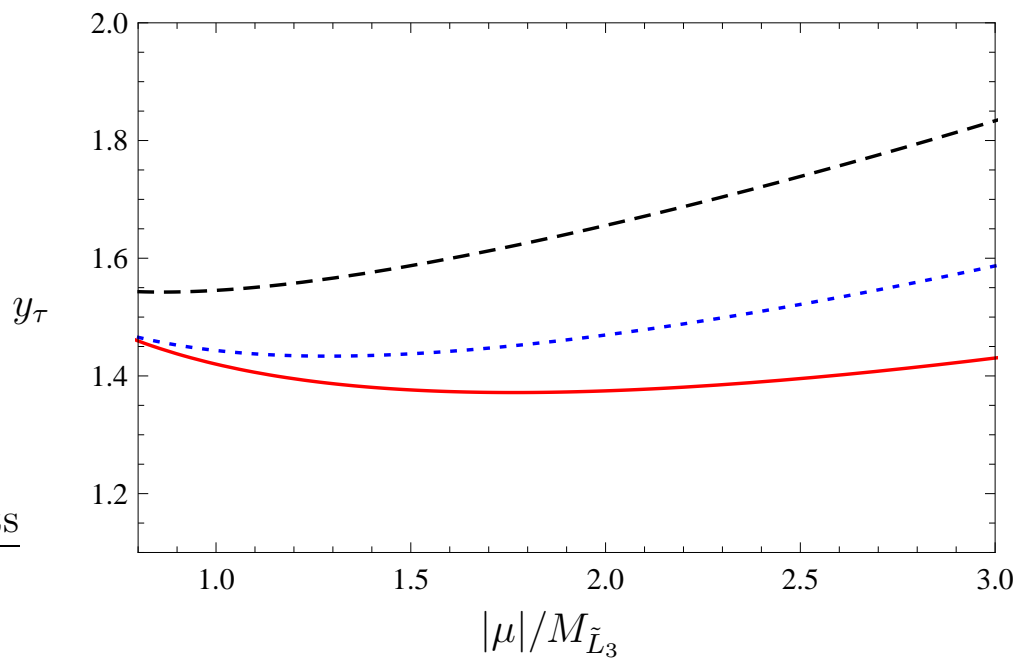

Figure 3: Tau Yukawa coupling to $H_{d}$ required to generate the correct $m_{\tau}$, for three values of $M_{\tilde{B}} / M_{\tilde{L}_{3}}: 0.3$ (dashed black line), 0.6 (dotted blue line), and 1 (solid red line). The other parameters are fixed as follows: $M_{\tilde{\tau}^{c}}=M_{\tilde{B}}, M_{\tilde{W}}$ as in Eq. (2.18), $\tan \beta=200$, $\theta_{\mu}+\theta_{W}=\pi$, and $\theta_{W}=\theta_{B}$.

The resulting lepton mass is given by

$$
m_{\ell}=y_{\ell} v_{d}+y_{\ell}^{\prime} v_{u}
$$

We assume throughout that the squark and slepton mass matrices are proportional to the identity matrix in flavor space, so that their exchange in loops does not introduce additional flavor violation. Universal squark and slepton masses would arise, for instance, in gauge mediation [6]. Both $y_{\ell}$ and $y_{\ell}^{\prime}$ are $3 \times 3$ matrices in flavor space, with $y_{\ell}^{\prime} \propto y_{\ell}$. Using a global $S U(3)$ transformation, we can take these matrices to be diagonal, so that the physical masses of the charged leptons are

$$
\left\{m_{e}, m_{\mu}, m_{\tau}\right\}=\left\{\left|y_{\ell 11} v_{d}+y_{\ell 11}^{\prime} v_{u}\right|,\left|y_{\ell 22} v_{d}+y_{\ell 22}^{\prime} v_{u}\right|,\left|y_{\ell 33} v_{d}+y_{\ell 33}^{\prime} v_{u}\right|\right\}
$$

It is unlikely that the ratio $|\mu| / M_{\tilde{e}}$ is much larger than unity because the lower limit on the charged slepton masses is of order $100 \mathrm{GeV}$, and the $|\mu|$ parameter cannot be higher than the electroweak scale without fine-tuning. This, in conjunction with the limit (3.4) implies that, in order to obtain a sufficiently large $\tau$ mass, it is necessary for the $\left(y_{\ell}\right)_{33} \equiv y_{\tau}$ Yukawa coupling to be above some value of order 1. For example, when the gaugino masses satisfy the unification condition of Eq. (2.18), the complex phases vanish, and we set $M_{\tilde{\tau}^{c}} \approx M_{\tilde{B}}$ for simplicity, we find $y_{\tau} \gtrsim 1$ for $|\mu| \lesssim 3 M_{\tilde{L}_{3}}$ (see Figure 3). Although this Yukawa coupling is large, it is still perturbative. 

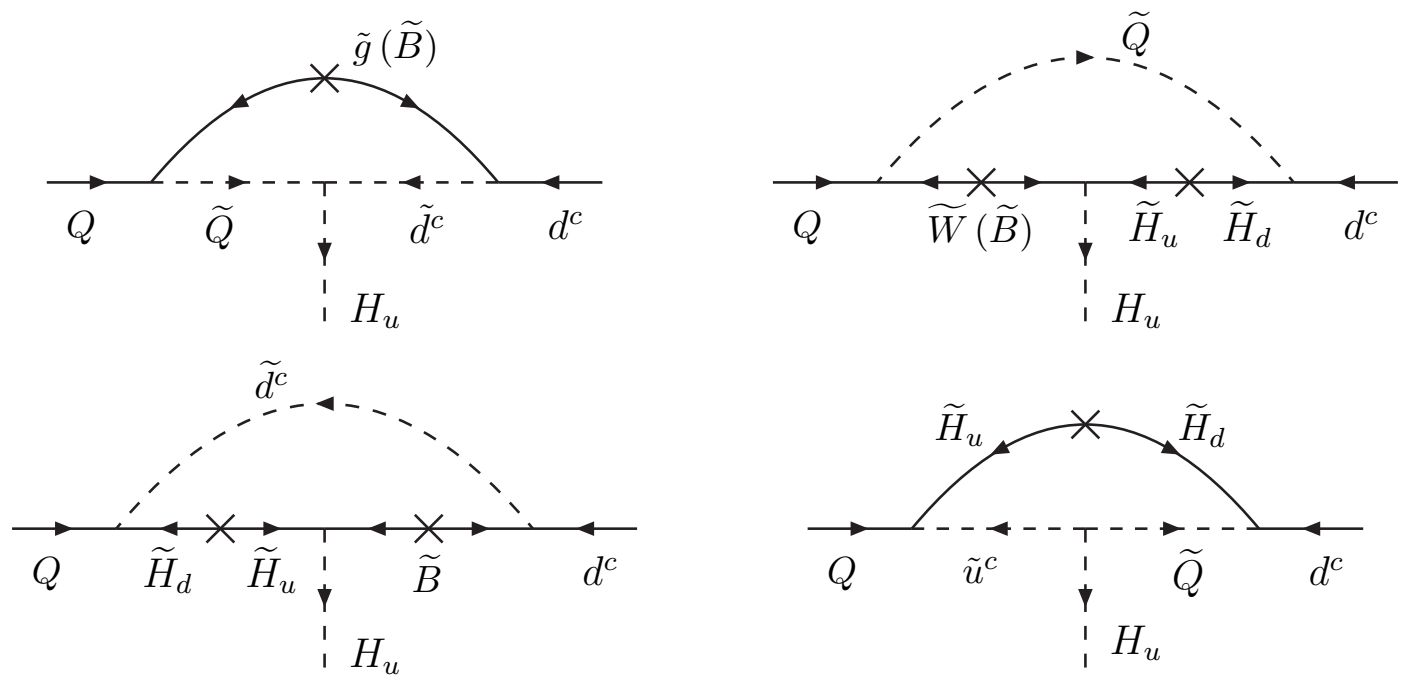

Figure 4: Diagrams responsible for the down-type quark masses. The first diagram involves the $F$-term interaction given in Eq. (2.9). The next two diagrams involve the gaugino interactions of $H_{u}$ given in Eq. (2.11). The last diagram relies on the supersymmetrybreaking trilinear term (2.12).

\section{Loop-induced down-type quark masses}

We now turn to the 1-loop diagrams which contribute to the $y_{d}^{\prime}$ Yukawa coupling of the down-type quarks to $H_{u}^{\dagger}$. Compared to the lepton case, there are more diagrams (see Figure 4). The F-term interaction for quarks given in Eq. (2.9) appears in a loop that involves either a bino (as in the case of leptons) or a gluino. The ensuing uplifted-Higgs coupling is given by

$$
\left(y_{d}^{\prime}\right)_{F}=-\frac{y_{d}}{3 \pi} e^{-i\left(\theta_{g}+\theta_{\mu}\right)} \frac{2|\mu|}{M_{\tilde{d}}}\left[\alpha_{s} F\left(\frac{M_{\tilde{g}}}{M_{\tilde{Q}}}, \frac{M_{\tilde{d}}}{M_{\tilde{Q}}}\right)+\frac{\alpha e^{i\left(\theta_{g}-\theta_{B}\right)}}{24 c_{W}^{2}} F\left(\frac{M_{\tilde{B}}}{M_{\tilde{Q}}}, \frac{M_{\tilde{d}}}{M_{\tilde{Q}}}\right)\right]
$$

The gaugino interactions of Eq. (2.11) induce the same contributions as in the lepton sector except for the replacement of sleptons by squarks:

$\left(y_{d}^{\prime}\right)_{\tilde{H}}=-\frac{y_{d} \alpha}{8 \pi} e^{-i\left(\theta_{W}+\theta_{\mu}\right)}\left\{\frac{3}{s_{W}^{2}} F\left(\frac{M_{\tilde{W}}}{M_{\tilde{Q}}}, \frac{|\mu|}{M_{\tilde{Q}}}\right)+\frac{e^{i\left(\theta_{W}-\theta_{B}\right)}}{3 c_{W}^{2}}\left[F\left(\frac{M_{\tilde{B}}}{M_{\tilde{Q}}}, \frac{|\mu|}{M_{\tilde{Q}}}\right)+2 F\left(\frac{M_{\tilde{B}}}{M_{\tilde{d}}}, \frac{|\mu|}{M_{\tilde{d}}}\right)\right]\right\}$.

There is also a novel type of contribution to $y_{d}^{\prime}$ coming from the supersymmetry-breaking trilinear term of Eq. (2.12), shown in the last diagram of Figure 4. The source of Rsymmetry breaking in this case is the scalar $A$ term. This contribution to the upliftedHiggs coupling of the down-type quarks is

$$
\left(y_{d}^{\prime}\right)_{A}=-\frac{y_{u} y_{d}}{16 \pi^{2}} e^{-i \theta_{\mu}} \frac{A_{u}^{*}}{M_{\tilde{u}}} F\left(\frac{M_{\tilde{u}}}{M_{\tilde{Q}}}, \frac{|\mu|}{M_{\tilde{Q}}}\right) \text {. }
$$




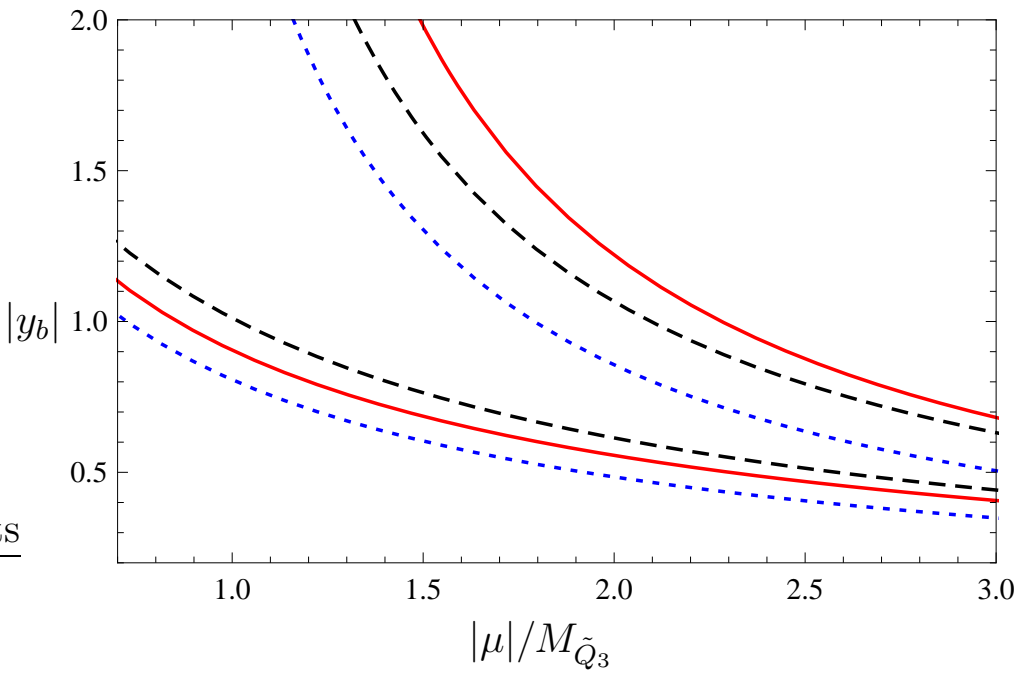

Figure 5: Absolute value of the bottom Yukawa coupling to $H_{d}$ required to generate the correct $m_{b}$, for three values of $M_{\tilde{B}} / M_{\tilde{Q}_{3}}: 0.1$ (dashed black lines), 0.5 (dotted blue lines), and 1 (solid red lines). The upper (lower) set of lines corresponds to $\theta_{g}=-\theta_{\mu}$ $\left(\theta_{g}=\pi-\theta_{\mu}\right)$. The other parameters are fixed as follows: $M_{\tilde{Q}}=M_{\tilde{b}^{c}}$, the gaugino masses satisfy the unification condition, $\tan \beta=200, A_{t}=0, \theta_{B}=\theta_{W}$ and $\theta_{\mu}+\theta_{W}=\pi$.

The effective Yukawa coupling of $H_{u}^{\dagger}$ to down-type quarks is then the sum of the contributions shown in Figure 4 .

$$
y_{d}^{\prime}=\left(y_{d}^{\prime}\right)_{F}+\left(y_{d}^{\prime}\right)_{\tilde{H}}+\left(y_{d}^{\prime}\right)_{A}
$$

The dominant contribution to the effective Yukawa typically comes from the first term in (4.1), although there is sensitivity to the details of the superpartner spectrum and the other diagrams may be comparable in certain regimes. For simplicity we assume that the gaugino masses obey the unification relations of Eq. (2.18) and

$$
M_{\tilde{g}}=M_{\tilde{W}} \frac{\alpha_{s}}{\alpha} s_{W}^{2}
$$

that $M_{\tilde{Q}}=M_{\tilde{b}^{c}}$ and that the $A$ terms are negligible, and we show in Figure 5 the $y_{b}$ coupling necessary to generate the correct bottom quark mass. The value of $y_{b}$ is sensitive to the relative phase between the wino and gluino masses because of the cancellation between the first terms of Eqs. (4.1) and (4.2). As mentioned in Section 3, we expect an upper bound on $\mu$ of order the electroweak scale, leading to a lower limit on $y_{b}$. The requirement of a perturbative Yukawa coupling places an upper bound on the squark masses. Furthermore, for light squarks the Yukawa coupling to $H_{d}$ is often larger for the tau than for the bottom quark. Note that even in the context of gauge mediation, 
the relations between the squark and slepton masses depend on physics at scales much above the TeV scale [7], so that the $x$ axes in Figures 3 and 5 cannot be straightforwardly compared.

\section{Properties of the uplifted Higgs states}

In the presence of the loop-induced $H_{u} H_{d}$ soft term, the neutral CP-even Higgs states mix with a very small angle $\alpha$ which satisfies

$$
\tan \alpha=-\left(\frac{M_{A^{0}}^{2}+M_{Z}^{2}}{M_{A^{0}}^{2}-M_{Z}^{2}}\right) \frac{1}{\tan \beta}\left[1+O\left(1 / \tan ^{2} \beta\right)\right] .
$$

In deriving this relation we used the phenomenological requirement that the $A^{0}$ boson is significantly heavier than the $Z$ boson. This mixing shifts the $H^{0}$ mass upwards by a small amount:

$$
M_{H^{0}}^{2} \simeq M_{A^{0}}^{2}\left(1+\frac{4 M_{Z}^{2}}{\left(M_{A^{0}}^{2}-M_{Z}^{2}\right) \tan ^{2} \beta}\right),
$$

where we ignored the terms suppressed by more powers of $\tan \beta$. The mass of $h^{0}$ is pushed downward by the mixing, but in addition there is the usual positive contribution from 1-loop corrections to the quartic terms in the Higgs potential:

$$
M_{h^{0}}^{2} \simeq M_{Z}^{2}\left(1-\frac{4 M_{A}^{2}}{\left(M_{A^{0}}^{2}-M_{Z}^{2}\right) \tan ^{2} \beta}\right)+\Delta\left(M_{h^{0}}^{2}\right)
$$

The relations between $M_{A^{0}}, M_{H^{ \pm}}$and the mass parameters in the Lagrangian given in Eq. (2.6) remain valid even in the presence of the $H_{u} H_{d}$ soft term.

The heavy Higgs states couple to the $b$ quark as follows

$$
y_{H^{0}}^{b} H^{0} \bar{b} b+y_{A^{0}}^{b} A^{0} \bar{b} \gamma_{5} b+\left(y_{H^{-}}^{b} H^{-} \bar{b}_{R} t_{L}+y_{H^{-}}^{t} H^{-} \bar{b}_{L} t_{R}+\text { H.c. }\right)
$$

where the Yukawa couplings are given by

$$
\begin{aligned}
& y_{H^{0}}^{b}=-\frac{1}{\sqrt{2}}\left(y_{b} \cos \alpha+y_{b}^{\prime} \sin \alpha\right) \approx-\frac{y_{b}}{\sqrt{2}}, \\
& y_{A^{0}}^{b}=y_{H^{-}}^{b}=\frac{1}{\sqrt{2}}\left(y_{b} \sin \beta-y_{b}^{\prime} \cos \beta\right) \approx \frac{y_{b}}{\sqrt{2}}, \\
& y_{H^{-}}^{t}=\frac{1}{\sqrt{2}} y_{t}^{*} \cos \beta \approx \frac{m_{t}}{\sqrt{2} v_{u} \tan \beta} .
\end{aligned}
$$


Given that $\tan \beta$ is so large, the first three of the above couplings are essentially determined by the tree level Yukawa coupling of the $H_{d}$ doublet. Analogous expressions describe the couplings of the heavy Higgs states to the $s$ and $d$ quarks, as well as to the charged leptons. Thus, in the uplifted Higgs region, the Yukawa couplings of the heavy Higgs states are substantially larger than in the usual MSSM. Consequently the heavy Higgs states are wider, and their branching fractions are altered when compared to the MSSM. In particular, the branching fractions to tau leptons, which are given at tree level by

$$
B\left(H^{0}, A^{0} \rightarrow \tau^{+} \tau^{-}\right) \approx \frac{y_{\tau}^{2}}{y_{\tau}^{2}+3 y_{b}^{2}}
$$

are enhanced compared to the usual MSSM (where it is about $10 \%$ at tree leve11), and in some regions of parameter space can be larger than $80 \%$. Similarly, the Higgsino decays are altered.

The $h^{0}$ boson has a Yukawa coupling to the $b$ quark given by

$$
\begin{aligned}
y_{h^{0}}^{b} & =\frac{1}{\sqrt{2}}\left(y_{b} \sin \alpha-y_{b}^{\prime} \cos \alpha\right) \\
& \approx-\frac{1}{\sqrt{2}}\left[\frac{y_{b}}{\tan \beta}\left(\frac{M_{A^{0}}^{2}+M_{Z}^{2}}{M_{A^{0}}^{2}-M_{Z}^{2}}\right)+y_{b}^{\prime}\right]\left[1+O\left(1 / \tan ^{2} \beta\right)\right] .
\end{aligned}
$$

It is interesting that in the uplifted Higgs region the $h^{0}$ couplings to a quark or lepton depend on both the tree level Yukawa coupling to that fermion, $y_{f}$, as well as on the loop-generated Yukawa coupling $y_{f}^{\prime}$. Note that in the decoupling limit, where $M_{A} \rightarrow \infty$, the first term in the above expression vanishes because $\tan \beta \rightarrow \infty$ [see Eq. (2.17)] while $y_{b}$ is bounded from above. However, the decoupling limit is approached relatively slowly: e.g., for $M_{A}=700 \mathrm{GeV}, y_{b} \approx 1$ and $\tan \beta \approx 100$, the $h^{0} \bar{b} b$ coupling differs from $y_{b}^{\prime}$, which is the standard model value, by $50 \%$. By contrast, in the usual MSSM the decoupling limit is approached faster: for $M_{A}$ as small as $300 \mathrm{GeV}$, the $h^{0} \bar{b} b$ coupling is essentially equal to the standard model one [8].

\section{Outlook}

We have shown that the MSSM includes a significant region of parameter space which is viable and has not been previously explored. In this 'uplifted' region, only $H_{u}$ has

\footnotetext{
${ }^{1}$ Loop corrections to the Yukawa couplings of the heavy Higgs particles can make the branching fractions to taus as large as $25 \%$ for $\tan \beta=30$ [ $]$.
} 
a tree-level VEV, and the down-type fermions get masses mostly from 1-loop induced couplings to $H_{u}$. Assuming that the coefficient of the $H_{u} H_{d}$ soft term vanishes at the supersymmetry-breaking scale, its value at the weak scale induced by MSSM fields at 1loop gives rise to a tiny $\mathrm{VEV}$ for the $H_{d}$ doublet. Thus, $\tan \beta=v_{u} / v_{d} \gtrsim 100$ at the weak scale, but the Yukawa couplings of the down-type fermions to $H_{d}$ remain perturbative for a range of parameters (see Figures 3 and 5 ). In fact $\tan \beta$ can be a confusing parameter, as the usual relations between fermion masses and couplings to the heavy Higgs states do not apply in the uplifted region. In particular, the ratio of the tree-level couplings of $H^{0}$ (or $A^{0}$ ) to $\bar{b} b$ and $\tau^{+} \tau^{-}$is no longer fixed as in the usual MSSM, and the branching fraction for $H^{0}, A^{0} \rightarrow \tau^{+} \tau^{-}$may be the dominant one within the uplifted region.

The Yukawa couplings of the $b$ quark to the heavy Higgs states is large, of order unity, leading to several implications for phenomenology. First, there are enhanced flavor changing processes such as $b \rightarrow s \gamma$ and $b \rightarrow s \ell^{+} \ell^{-}$, similar to those in the usual MSSM at $\tan \beta \approx 30[9]$ because that corresponds to $y_{b} \sim 1$. More importantly, the production of $H^{0}$ and $A^{0}$ at the Tevatron and LHC is large, mainly in association with a $b \bar{b}$ pair, but also through gluon fusion induced by a $b$ loop (as well as loops involving $\tilde{b}$ squarks) [10]. This, in conjunction with the large Yukawa coupling of the $\tau$ lepton to the heavy Higgs bosons, implies that the $A^{0}$ and $H^{0}$ particles can be discovered in the $\tau^{+} \tau^{-} b \bar{b}$ or $\tau^{+} \tau^{-}$ final states. Likewise, the $H^{ \pm}$particle can be copiously produced in association with a $b \bar{t}$ or $\bar{b} t$ pair and would have a high decay rate into $\tau \nu$. We leave the detailed study of the phenomenological implications at hadron colliders for a future publication.

The Yukawa coupling of the heavy Higgs particles to muons are also enhanced compared to the standard model Higgs coupling by two orders of magnitude. As a result, $s$-channel production of $A^{0}$ and $H^{0}$ at a muon collider followed by the decay into $\tau^{+} \tau^{-}$ would be an excellent way of studying the uplifted Higgs region.

Besides predicting an unusual Higgs phenomenology, the uplifted region has the merit of explaining the smallness of $m_{\tau} / m_{t}$ and $m_{b} / m_{t}$ in terms of a loop factor. It would be interesting to extend this explanation within the MSSM to the masses of the second and first generation fermions, perhaps along the lines of the domino mechanism [11]. 


\section{A Appendix}

There are several classes of diagrams contributing to the generation of uplifted couplings all involve three internal propagators, thus it is useful to define a function

$$
\int \frac{d^{4} k}{(2 \pi)^{4}} \frac{i}{\left(k^{2}-m_{1}^{2}\right)\left(k^{2}-m_{2}^{2}\right)\left(k^{2}-m_{3}^{2}\right)}=\frac{1}{16 \pi^{2}} \frac{1}{m_{2} m_{3}} F\left(\frac{m_{2}}{m_{1}}, \frac{m_{3}}{m_{1}}\right)
$$

the form of $F$ was given in (3.2). The diagram involving an internal gluino line (the left-hand diagram in the first row of Figure 4) gives a contribution to $-i y_{d}^{\prime} H_{u}^{*} Q d^{c}$ of

$$
\begin{gathered}
\int \frac{d^{4} k}{(2 \pi)^{4}}\left(-i \sqrt{2} e^{-i \frac{\theta_{g}}{2}} g_{3} t^{a}\right)\left(-i \sqrt{2} e^{-i \frac{\theta_{g}}{2}} g_{3} t^{a}\right)\left(i \mu^{*} y_{d}\right) \frac{i}{k^{2}-M_{\tilde{Q}}^{2}} \frac{i}{k^{2}-M_{\tilde{d}}^{2}} \frac{i M_{\tilde{g}}}{k^{2}-M_{\tilde{g}}^{2}} \\
=i \frac{\alpha_{s}}{4 \pi} \frac{8}{3} \frac{|\mu|}{M_{\tilde{d}}} e^{-i\left(\theta_{g}+\theta_{\mu}\right)} y_{d} F\left(\frac{M_{\tilde{g}}}{M_{\tilde{Q}}}, \frac{M_{\tilde{d}}}{M_{\tilde{Q}}}\right) \cdot
\end{gathered}
$$

Thus the contribution to $y_{d}^{\prime}$ is

$$
-\frac{\alpha_{s}}{4 \pi} \frac{8}{3} \frac{|\mu|}{M_{\tilde{d}}} e^{-i\left(\theta_{g}+\theta_{\mu}\right)} y_{d} F\left(\frac{M_{\tilde{g}}}{M_{\tilde{Q}}}, \frac{M_{\tilde{d}}}{M_{\tilde{Q}}}\right),
$$

and there is a similar contribution from diagrams involving an internal Bino, but not a Higgsino, (the bottom diagram of Figure 2 and the left-hand diagram in the first row of Figure 4) where the $S U(3)$ coupling and group generators are replaced with those for $U(1)$.

The Wino diagram (the left-hand diagram of Figure 2 and the right-hand diagram in the first row of Figure (4) is most easily evaluated in $S U(2)_{L}$ components. The diagram with charged Higgsinos on the internal line gives,

$$
\begin{gathered}
\int \frac{d^{4} k}{(2 \pi)^{4}}\left(-i \sqrt{2} e^{-i \frac{\theta_{W}}{2}} g_{2} \cdot \frac{1}{\sqrt{2}}\right)\left(-i \sqrt{2} e^{-i \frac{\theta_{W}}{2}} g_{2} \cdot \frac{1}{\sqrt{2}}\right)\left(i y_{\ell}\right) \frac{i M_{\tilde{W}}}{k^{2}-M_{\tilde{W}}^{2}} \frac{i \mu^{*}}{k^{2}-|\mu|^{2}} \frac{i}{k^{2}-M_{\tilde{L}}^{2}} \\
=i \frac{\alpha_{2}}{4 \pi} e^{-i\left(\theta_{W}+\theta_{\mu}\right)} y_{\ell} F\left(\frac{M_{\tilde{W}}}{M_{\tilde{L}}}, \frac{|\mu|}{M_{\tilde{L}}}\right) .
\end{gathered}
$$

The same diagram but with neutral Higgsinos is,

$$
\begin{gathered}
\int \frac{d^{4} k}{(2 \pi)^{4}}\left(-i \sqrt{2} e^{-i \frac{\theta_{W}}{2}} g_{2} \cdot-\frac{1}{2}\right)\left(-i \sqrt{2} e^{-i \frac{\theta_{W}}{2}} g_{2} \cdot-\frac{1}{2}\right)\left(-i y_{\ell}\right) \frac{i M_{\tilde{W}}}{k^{2}-M_{\tilde{W}}^{2}} \frac{-i \mu^{*}}{k^{2}-|\mu|^{2}} \frac{i}{k^{2}-M_{\tilde{L}}^{2}} \\
=i \frac{\alpha_{2}}{4 \pi} \frac{1}{2} e^{-i\left(\theta_{W}+\theta_{\mu}\right)} y_{\ell} F\left(\frac{M_{\tilde{W}}}{M_{\tilde{L}}}, \frac{|\mu|}{M_{\tilde{L}}}\right) .
\end{gathered}
$$


Note the additional minus signs in the Yukawa coupling and the Higgsino mass in the propagator, both due to the contraction with $\epsilon^{a b}$ in the definition of the Lagrangian terms. In addition the gauge couplings are due to $\tau^{ \pm}$for the charged Higgsino case and $\tau^{3}$ for the neutral. Thus, the contribution to $y_{\ell}^{\prime}$ is

$$
-\frac{3 \alpha_{2}}{8 \pi} e^{-i\left(\theta_{W}+\theta_{\mu}\right)} y_{\ell} F\left(\frac{M_{\tilde{W}}}{M_{\tilde{L}}}, \frac{|\mu|}{M_{\tilde{L}}}\right) .
$$

Diagrams involving a Bino and Higgsino give similar results.

\section{References}

[1] For a review see, S. P. Martin, "A Supersymmetry Primer," arXiv:hep-ph/9709356.

[2] L. J. Hall, R. Rattazzi and U. Sarid, "The Top quark mass in supersymmetric SO(10) unification," Phys. Rev. D 50, 7048 (1994) arXiv:hep-ph/9306309;

T. Blazek, S. Raby and S. Pokorski, "Finite supersymmetric threshold corrections to CKM matrix elements in the large $\tan \beta$ regime," Phys. Rev. D 52, 4151 (1995) arXiv:hep-ph/9504364;

D. M. Pierce, J. A. Bagger, K. T. Matchev and R. j. Zhang, "Precision corrections in the MSSM," Nucl. Phys. B 491, 3 (1997) arXiv:hep-ph/9606211];

F. Borzumati, G. R. Farrar, N. Polonsky and S. D. Thomas, "Soft Yukawa couplings in supersymmetric theories," Nucl. Phys. B 555, 53 (1999) arXiv:hep-ph/9902443;

H. E. Haber and J. D. Mason, "Hard supersymmetry-breaking 'wrong-Higgs' couplings of the MSSM," Phys. Rev. D 77, 115011 (2008) [arXiv:0711.2890 [hep-ph]];

M. Beneke, P. Ruiz-Femenia, M. Spinrath, "Higgs couplings in the MSSM at large tan(beta)," JHEP 0901, 031 (2009) arXiv:0810.3768 [hep-ph]];

M. Gorbahn, S. Jager, U. Nierste et al., "The supersymmetric Higgs sector and BBbar mixing for large tan beta," [arXiv:0901.2065 [hep-ph]].

[3] C. Hamzaoui and M. Pospelov, "Up-down unification just above the supersymmetric threshold," Eur. Phys. J. C 8, 151 (1999) arXiv:hep-ph/9803354;

C. Hamzaoui, M. Pospelov and M. Toharia, "Higgs-mediated FCNC in supersymmetric models with large tan $\beta$," Phys. Rev. D 59, 095005 (1999) arXiv:hep-ph/9807350.

[4] M. S. Carena, S. Heinemeyer, C. E. M. Wagner and G. Weiglein, "MSSM Higgs boson searches at the Tevatron and the LHC: Impact of different benchmark scenarios," Eur. Phys. J. C 45, 797 (2006) arXiv:hep-ph/0511023. 
[5] S. Nandi and Z. Tavartkiladze, "A New Extensions of MSSM: FMSSM," Phys. Lett. B 672, 240 (2009) arXiv:0804.1996 [hep-ph]].

[6] M. Dine, A. E. Nelson and Y. Shirman, "Low-Energy Dynamical Supersymmetry Breaking Simplified," Phys. Rev. D 51, 1362 (1995) arXiv:hep-ph/9408384]; M. Dine, A. E. Nelson, Y. Nir and Y. Shirman, "New tools for low-energy dynamical supersymmetry breaking," Phys. Rev. D 53, 2658 (1996) arXiv:hep-ph/9507378.

[7] S. P. Martin, "Generalized messengers of supersymmetry breaking and the sparticle mass spectrum," Phys. Rev. D 55, 3177 (1997) arXiv:hep-ph/9608224;

M. Dine, Y. Nir and Y. Shirman, "Variations on minimal gauge mediated supersymmetry breaking," Phys. Rev. D 55, 1501 (1997) arXiv:hep-ph/9607397];

P. Meade, N. Seiberg and D. Shih, "General Gauge Mediation," Prog. Theor. Phys. Suppl. 177, 143 (2009) [arXiv:0801.3278 [hep-ph]].

[8] For a review, see M. S. Carena and H. E. Haber, "Higgs boson theory and phenomenology," Prog. Part. Nucl. Phys. 50, 63 (2003) arXiv:hep-ph/0208209];

H. E. Haber, H. E. Logan, S. Penaranda and D. Temes, "Quantum corrections to the MSSM $h^{0} b \bar{b}$ vertex: Decoupling limit," Nucl. Phys. Proc. Suppl. 157, 162 (2006) arXiv:hep-ph/0601237.

[9] K. S. Babu and C. F. Kolda, "Higgs mediated $B^{0} \rightarrow \mu^{+} \mu^{-}$in minimal supersymmetry," Phys. Rev. Lett. 84, 228 (2000) arXiv:hep-ph/9909476;

M. S. Carena, D. Garcia, U. Nierste and C. E. M. Wagner, " $b \rightarrow s \gamma$ and supersymmetry with large tan $\beta$," Phys. Lett. B 499, 141 (2001) arXiv:hep-ph/0010003;

A. J. Buras, P. H. Chankowski, J. Rosiek and L. Slawianowska, " $\Delta M_{d, s}, B^{0} d, s \rightarrow$ $\mu^{+} \mu^{-}$and $B \rightarrow X_{s} \gamma$ in supersymmetry at large $\tan \beta$," Nucl. Phys. B 659, 3 (2003) arXiv:hep-ph/0210145;

M. S. Carena, A. Menon, R. Noriega-Papaqui, A. Szynkman and C. E. M. Wagner, "Constraints on B and Higgs physics in minimal low energy supersymmetric models," Phys. Rev. D 74, 015009 (2006) [arXiv:hep-ph/0603106].

[10] For a review see, A. Djouadi, "The Anatomy of electro-weak symmetry breaking. II. The Higgs bosons in the minimal supersymmetric model," Phys. Rept. 459, 1 (2008) arXiv:hep-ph/0503173. 
[11] B. A. Dobrescu and P. J. Fox, "Quark and lepton masses from top loops," JHEP 0808, 100 (2008) [arXiv:0805.0822 [hep-ph]];

P. W. Graham and S. Rajendran, "A Domino Theory of Flavor," arXiv:0906.4657, 